\title{
DETERMINAÇÃO DE CARBONO ORGÂNICO EM POVOAMENTOS DE Acacia mearnsii DE WILD. PLANTADOS NO RIO GRANDE DO SUL
}

\section{Determination of organic carbon in stands of Acacia mearnsii de Wild. planted in the state Rio Grande do Sul}

\author{
Marcos Vinicius Winckler Caldeira \\ Mauro Valdir Schumacher" \\ Leonir Rodrigues Barichello \\ Hamilton Luiz Munari Vogel
}

\section{Resumo}

O presente estudo teve como objetivo determinar a quantidade de carbono orgânico no solo, serapilheira e biomassa, em povoamentos de Acacia mearnsii, com 4 e 6 anos de idade. O estudo foi realizado na Empresa Florestal AGROSETA S.A., localizada no município de Butiá-RS. Em cada uma das idades do povoamento, a ser amostrado o carbono orgânico no solo, foram abertas três trincheiras, com $100 \mathrm{~cm}$ de profundidade. Em cada uma destas, a amostragem foi feita a cada $20 \mathrm{~cm}$, onde amostras foram coletadas para determinação da densidade do solo e análise do carbono orgânico no solo. As coletas de serapilheira foram realizadas com o auxílio de uma moldura de ferro, com dimensões de $0,25 \mathrm{~m}$ x 0,25 m, de forma aleatória, com 24 repetições por idade. Para determinação da biomassa em cada uma das idades ( 4 e 6 anos), inicialmente, foram levantados os diâmetros de seis parcelas de $18 \mathrm{~m}$ x $24 \mathrm{~m}$, e posterior distribuição diamétrica, dividindo em três classes e abatendo a árvore de diámetro médio em cada classe. Concluiu-se que o estoque de carbono orgânico no solo até $100 \mathrm{~cm}$ de profundidade foi de 110,1 Mg.ha- ${ }^{-1}$ e 101,5 Mg.ha' ${ }^{-1}$ para o povoamento de A. mearnsii, com 4 e 6 anos de idade, respectivamente; para o povoamento de $A$. mearnsii, com 4 anos de idade, o estoque de carbono orgânico foi de 2,26 Mg.ha-1 e 27,68 Mg.ha-1, na serapilheira e biomassa total, respectivamente, sendo que $17,1 \mathrm{Mg} \mathrm{ha}^{-1}$ do carbono orgânico estão armazenados na madeira, porém, para o povoamento, com 6 anos de idade, o estoque de carbono orgânico foi de 4,53 Mg. ha ${ }^{-1}$ e 55,75 Mg.ha', na serapilheira e biomassa total, respectivamente, com 36,6 Mg.ha-1 do carbono orgânico retidos na madeira.

Palavras-chave: Carbono, Acacia mearnsii, Biomassa.

\footnotetext{
* Doutorando em Ciências Florestais, Centro de Ciências Florestais e da Madeira/UFPR. Av. Pref. ${ }^{\circ}$ Lothário Meissner, n. ${ }^{\circ}$ 3400, Jardim Botânico, Curitiba - PR, CEP 80210-170. E-mail: caldeira@floresta.ufpr.br

** Prof. ${ }^{\circ}$ Dr. do Departamento de Ciências Florestais, CCR/UFSM. Bolsita do CNPq. schuma@ccr.ufsm.br.

*** Pós-graduando em Ciências Florestais, CCR/UFSM
} 


\section{Abstract}

The present study had as objective to estimate the amount of organic carbon in the soil, litter and biomass, from Acacia mearnsii stands, with 4 and 6 years old. It was accomplished in AGROSETA S.A., located in Butiá county, RS. In each age of the forest, where the organic carbon was sampled, three ditches were trenched with $100 \mathrm{~cm}$ deep. In each one of this, the sampling was done in each $20 \mathrm{~cm}$, where samples to determine tbc density of the soil and analysis of the soil organic carbon were collected. The litter assessments were done with the assistance of an iron frame with 0,25 $\mathrm{m} \times 0,25$ $m$ of dimensions, in a randomized way, with 24 repetitions per age. To the determination of the biomass in each stand with ages ( 4 and 6 years), the diameters were initially surveyed from six plots of $18 \mathrm{~m} \times 24 \mathrm{~m}$, and after, the diametric distributions were done, dividing in three classes and cuting the tree with the average diameter in each class. With this results, it is concluded that, the storage of organic carbon in the soil until $100 \mathrm{~cm}$ deep was the $110,1 \mathrm{Mg} \mathrm{ha}^{-1}$ and $101,5 \mathrm{Mg} \mathrm{ha}^{-1}$ to the stand of $A$. mearnsii with 4 and 6 years of age, respectively; to the $A$. mearnsii stand with 4 years, the storage of organic carbon was the $226 \mathrm{Mg} \mathrm{ha}^{-1}$ and 27,68 $\mathrm{Mg} \mathrm{ha}^{-1}$, on the litter and total biomass, respectively, beeing $17,1 \mathrm{Mg} \mathrm{ha}^{-1}$ from the organic carbon stored an the wood; to the stand with 6 years, the storage of organic carbon was 4,53 $\mathrm{Mg} \mathrm{ha}^{-1}$ and 55,75 $\mathrm{Mg} \mathrm{ha}^{-1}$, an the litter and total biomass, respectively, with $36,6 \mathrm{Mg} \mathrm{ha}^{-1}$ of the organic carbon retained an the wood.

Key words: Carbon, Acacia mearnsii, Biomass.

\section{Introdução}

A conservação e ampliação das florestas naturais e plantadas, segundo Rochadelli (2001), tem sido aceita como de importância primordial no propósito de reduzir os níveis de gás carbônico na atmosfera. Todas as plantas absorvem gás carbônico e convertem em carboidratos na forma de tecidos da madeira, folhas, sementes e frutos.

As florestas plantadas, geralmente com fins econômicos e comerciais, são orientadas por critérios técnicos, conforme um plano de manejo, definindo-se a época de sua colheita. Porém, durante sua existência, estas florestas realizam a atividade de captura e fixação de carbono na madei- ra e demais componentes da biomassa. Assim, a quantificação do carbono orgânico torna-se importante, porque durante a existência destas florestas são imobilizadas quantidades de carbono existentes na atmosfera, principalmente na madeira.

A remoção do dióxido de carbono da atmosfera ocorre por meio da fotossíntese nos vegetais, e sua liberação pela respiração, sendo que parte deste carbono removido é armazenado na madeira. Medições na concentração de $\mathrm{CO}_{2}$ na atmosfera são menores durante o verão, quando ocorre maior atividade da fotossíntese, com maior crescimento dos vegetais, e maiores concentrações de $\mathrm{CO}_{2}$ são registradas no inverno, devido a menor fotossíntese realizada pelas plantas, com conseqiiente redução do crescimento (SIMPSON; BOTKIN, 1992).

Estudos de biomassa e de teor de carbono precisam ser estudados com mais frequência em diversas florestas e povoamentos florestais no Brasil, mas a limitação destes estudos está na dificuladade de se ter uma metodologia adequada para determinar, principalmente, biomassa radicial, além de ser um trabalho que envolve um grande número de pessoas e um custo muito alto. Neste sentido, o presente estudo teve como objetivo detreminar a quantidade de carbono orgânico no solo, serapilheira e biomassa em povoamento de Acacia mearnsii, com 4 e 6 anos de idade, plantados em Butiá, RS.

\section{Material e métodos}

O presente foi realizado na Empresa Florestal AGROSETA S.A., localizada no município de Butiá, com coordenadas $30^{\circ} 07^{\prime} 12^{\prime \prime}$ de latitude Sul e $51^{\circ} 57^{\prime} 45^{\prime \prime}$ de longitude Oeste de Greenwich e altitude de $35 \mathrm{~m}$ s.n.m. A cidade pertencente a região fisionômica natural do Estado do Rio Grande do Sul é denominada Serra do Sudeste. Segundo a calssificação climática de Koeppen, o clima da região é do tipo Cfa, subtropical, com temperatura média anual entre 18 e $19^{\circ} \mathrm{C}$ (MORENO, 1961). O solo da região em estudo é caracterizado como Argissolo Vermelho Escuro (EMBRAPA, 1999), textura argilosa, relevo ondulado e substrato granito.

Na ocasião da coleta dos dados foi realizado o inventério florestal nos povoamentos (Tabela 1). 
TABELA 1 - Resultados do inventário florestal realizado nos povoamentos de $A$. mearnsii Table 1 - Forest inventory results from stands of $A$. mearnsii

\begin{tabular}{ccccc}
\hline Idade (anos) & $\begin{array}{c}\text { Frequência } \\
\left(\text { árvores ha } \mathrm{ha}^{-1}\right)\end{array}$ & $\begin{array}{c}\text { DAP médio } \\
(\mathrm{cm})\end{array}$ & $\begin{array}{c}\text { Altura média } \\
(\mathrm{m})\end{array}$ & $\begin{array}{c}\text { Volume médio } \\
\left(\mathrm{m}^{3} \mathrm{ha}^{-1}\right)\end{array}$ \\
\hline 4 & 1995 & 8,1 & 10,7 & 81,54 \\
6 & 1690 & 10,9 & 13,4 & 135,87 \\
\hline
\end{tabular}

Em cada uma das idades do povoamento ( 4 e 6 anos), a ser amostrado o carbono orgânico no solo, foram abertas três trincheiras de $100 \mathrm{~cm}$ de profundidade. Em cada uma destas, a amostragem foi feita a cada $20 \mathrm{~cm}$, onde foram coletadas amostras para determinação da densidade do solo e análise do carbono orgânico no solo. A análise da densidade do solo foi realizada segundo o método do anel de Kopecky (EMBRAPA, 1997). O carbono orgânico no solo foi determinado, conforme a metodologia de Tedesco et al. (1995). O método utilizado foi o de Walkley-Black, com calor externo, descrito por Allison (1965), com aumento em $25 \%$ da concentração de dicromato, para maior abrangência de amostras com diferentes teores de $\mathrm{C}$ e decantação da fração mineral.

Nas trincheiras foram coletadas 5 amostras para densidade do solo e 5 para determinação do carbono, totalizando 75 amostras para análise física (densidade do solo) e 75 amostras para análise química (carbono orgânico). As análises foram realizadas no Laboratório de Ecologia Florestal do Departamento de Ciências Florestais da UFSM.

As coletas de serapilheira foram realizadas com o auxilio de uma moldura de ferro de $0,25 \mathrm{~m} \times 0,25 \mathrm{~m}$, de forma aleatória, com 24 repetições por idade. Após a coleta, o material foi devidamente acondicionado em sacos de papel e, posteriormente, levado ao Laboratório de Ecologia Florestal da UFSM. Neste, o material foi posto para secar em estufa de renovação e circulação de ar a uma temperatura média de $75^{\circ} \mathrm{C}$ até atingir peso constante. Após seco, o material foi resfriado em dissecadores e pesado em balança, com 0,01 g de precisão. O carbono orgânico da serapilheira também foi determinado de acordo com a metodologia de Tedesco et al. (1995).

Para as árvores de $A$. mearnsii, o carbono orgânico foi determinado nos seguintes componentes da biomassa: folhas, galhos vivos e mortos, casca, madeira e raiz.
Em cada uma das idades do povoamento, inicialmente, foram levantados os diâmetros de seis parcelas de $18 \mathrm{~m} \times 24 \mathrm{~m}$. Em seguida, foi realizado a distribuição diamétrica, dividindo em três classes. Após, foi feita a identificação da árvore de diâmetro médio em cada classe. Em seguida, as árvores foram abatidas e amostradas em folhas, galhos, madeira e casca, para determinação do percentual de umidade e posterior análise de carbono. Para amostragem de folhas, as mesmas foram coletadas do terço médio da copa e galhos vivos e galhos mortos, na parte intermediária. Para o tronco e casca, foi retirado um disco, $\operatorname{com} 5 \mathrm{~cm}$ de espessura a $50 \%$ da altura total da planta.

Após, foram removidos todos os galhos do tronco e, posteriormente, foram coletadas todas as folhas. Uma vez separadas às folhas dos galhos, ambos tiveram seu peso verde total aferido com balança de gancho e, também, o tronco foi separado em toretes, retirada a casca, também amostrados para análises de laboratório. Em seguida, foi quantificada a biomassa radicial das árvores. Para isto, foi necessário a demarcação da área útil de cada planta, para um espaçamento de 1,33 $\mathrm{m} \times 3$, m a área útil por planta é de 3,99 $\mathrm{m}^{2}$, a ser escavada até $100 \mathrm{~cm}$ de profundidade. As raízes foram arrancadas com o toco da árvore, separadas do mesmo, e, após, devidamente pesadas e amostradas para análise de laboratório. As análises de carbono orgânico no tecido vegetal foram feitas segundo metodologia proposta por Tedesco et al. (1995) no Laboratório de Ecologia Florestal da UFSM.

\section{Resultados e discussão}

Para ambos os povoamentos, houve um comportamento semelhante nos teores e quantidade de carbono orgânico no solo (Tabela 2). O maior teor e quantidader de carbono orgânico foi 
observado nos primeiros $20 \mathrm{~cm}$. Isso é em função do teor mais elevado de matéria orgânica (MO), nesta profundidade, proveniente principalmente da decomposição da serapilheira. Pode-se observar na Tabela 2 que os teores e quantidades de carbono orgânico diminuem na medida em que aumenta a profundidade do solo, pois esse fato é atribuído a redução dos teores de MO com a profundidade.

A quantidade média de carbono orgânico no solo varia muito em função do tipo de flo- resta ou povoamento e com a idade. Neste contexto, comparando os resultados do presente trabalho com o estudo realizado por Balbinot et al. (2000a), com Pinus taeda, com 15 e 20 anos de idade, observou-se que o carbono orgânico no solo, até $100 \mathrm{~cm}$ de profunidade, foi estimado em 246,0 e $189,7 \mathrm{Mg} \mathrm{ha}^{-1}$, respectivamente. Solos com plantios de pinus e eucaliptos mostram uma tendência de ter menos carbono orgânico (O'CONNELL ; SANKARAN, 1997).

TABELA 2 - Valores da densidade do solo (Ds), teores e quantidade de carbono orgânico no solo sob povoamentos de A. mearnsii, plantados no Rio Grande do Sul

Table 2 - Values of soil density (Ds), texts and amount of organic carbon in the ground under stands of $A$. mearnsii planted in Rio Grande do Sul

\begin{tabular}{cc|c|c|c|c|c}
\hline $\begin{array}{c}\text { Prof } \\
(\mathrm{cm})\end{array}$ & \multicolumn{6}{c}{ Ds } \\
& \multicolumn{2}{c}{$\left(\mathrm{g} \mathrm{cm}^{3}\right)$} & \multicolumn{2}{c}{$\mathrm{g} \mathrm{kg}^{-1}$} & \multicolumn{2}{c}{ Mg ha $^{-1}$} \\
\cline { 2 - 7 } & 4 anos & 6 anos & 4 anos & 6 anos & 4 anos & 6 anos \\
\hline $0-20$ & 1,47 & 1,37 & 9,1 & 10,0 & 26,1 & 27,3 \\
$20-40$ & 1,47 & 1,46 & 6,8 & 6,8 & 21,2 & 19,7 \\
$40-60$ & 1,44 & 1,43 & 8,9 & 7,8 & 25,6 & 22,5 \\
$60-80$ & 1,37 & 1,28 & 7,3 & 6,9 & 20,1 & 17,6 \\
$80-100$ & 1,31 & 1,42 & 6,4 & 5,1 & 16,6 & 14,3 \\
\hline Total & - & - & - & - & 109,6 & 101,4 \\
\hline
\end{tabular}

Conforme Schesinger (1977), a nível global, a quantidade média de carbono orgânico no solo florestal aumenta dos trópicos aos temperados, até as florestas boreais. Baixos valores de carbono orgânico no solo de florestas tropicais são causados por uma decomposição rápida, que compensa a produção ráida de serapilheira. A variação entre florestas tropicais pode ser muito grande, alcançando diferenças comparáveis entre tropicais e as florestas temperadas e boreais (ANDERSON \& SWIFT, 1983).

A distribuição de carbono orgânico, nos perfis dos solos, varia bastante a nível global, com menores quantidades armazenadas na superfície dos solos tropicais do que nos solos flo- restais de altitudes menores. Em média, de acordo com Schlesinger (1977), 1\% do carbono de um determinado perfil do solo é armazenado na camada superior da serapilheira nas florestas tropicais, enquanto que nas florestas boreais 13\%. Partindo deste princípio, ambos os povoamentos possuem quantidades bem semelhantes de carbono orgânico, armazenado na camada superior da serapilheira, ou seja, 1,1 e 1,0 $\mathrm{Mg} \mathrm{ha}^{-1}$ para 4 e 6 anos de idade, respectivamente (Tabela 2).

O teor, bem como a quantidade de carbono orgânico, é maior no povoamento de $A$. mearnsii, com 6 anos de idade (Tabela 3). Esse fato é ocasionado pela maior queda da serapilheira no ecossistema, em função do mai- 
or estádio de desenvolvimento deste povoamento. A variação na quantidade de serapilheira, acumulada nos solos florestais entre as diferentes plantações tropicais, expressa a influência dominante das características das espécies, idade dos povoamentos, taxa de incremento, con- dições climáticas e propriedadades do solo. Taxas de acumulação nos solos de plantações florestais podem variar significativamente entre diferentes espécies no mesmo sítio, conseqüentemente ocorre uma variação do carbono orgâniCO. (O'CONNELL; SANKARAN, 1997)

TABELA 3 - Biomassa média de serapilheira, teores e quantidades de carbono orgânico nos povoamentos de $A$. mearnssi, plantados no Rio Grande do Sul

Table 3 - Average biomass in the litter, texts and amounts of organic carbon in the A. mearnssi stands, planted in Rio Grande do Sul

\begin{tabular}{cccc}
\hline $\begin{array}{c}\text { Idade } \\
(\text { anos })\end{array}$ & $\begin{array}{c}\text { Serapilheira } \\
\mathrm{kg} \mathrm{ha}^{-1}\end{array}$ & \multicolumn{2}{c}{ Carbono orgánico } \\
\hline & & $\mathrm{g} \mathrm{ha}^{-1}$ & 2256,2 \\
4 & 7935,3 & 284,3 & 4532,9 \\
\hline
\end{tabular}

A quantidade de serapilheira acumulada e de carbono orgânico, no solo no povoamento com 6 anos de idade, foi bem superior em relação ao povoamento mais jovem (Tabela 3), além da idade, outros fatores podem estar influenciando, tais como: baixo nível de nutrientes na serapilheira e no solo; condições desfavoráveis para a decomposição, como déficit de água no solo e na serapilheira, temperaturas muito altas ou baixas, $\mathrm{pH}$ alto ou baixo; propriedades fisico-químicas da serapilheira como folhas, conteúdo de susbstâncias (ligninia, celulose, hemicelulose); baixa densida- de da população de organismos decompositores, além da época (estação do ano) da coleta da serapilheira acumulada. (O'CONNELL; SANKARAN, 1997)

Independente da idade do povoamento, as folhas, em relação a outros componentes da biomassa, possuem os maiores teores de carbono (Tabela 4). Isso não é somente uma característica do carbono, pois, determinados bioelementos, como N, P, K e Mg, Pereira e outros (2000) também apresentam os maiores teores nas folhas de A. mearnsii. 
TABELA 4 - Biomassa, teores e quantidade de carbono orgânico nos componentes da biomassa em povoamentos de A. mearnsii, plantados Rio Grande do Sul.

Table 4 - Biomass, texts and amount of organic carbon in the biomass components from A. mearnsii stands, planted in Rio Grande do Sul

\begin{tabular}{|c|c|c|c|}
\hline \multicolumn{4}{|c|}{ Povoamento com 4 anos de idade } \\
\hline \multirow[t]{2}{*}{ Componente } & \multirow[t]{2}{*}{ Biomassa $\left(\mathrm{kg} \mathrm{ha}^{-1}\right)$} & \multicolumn{2}{|c|}{ Carbono orgânico } \\
\hline & & $\mathrm{g} \mathrm{ha}^{-1}$ & $\mathrm{~kg} \mathrm{ha}^{-1}$ \\
\hline Folhas & 2547,9 & 433,7 & 1104,7 \\
\hline Galhos mortos & 2394,7 & 393,5 & 939,9 \\
\hline Galhos vivos & 7391,3 & 392,0 & 2897,5 \\
\hline Casca & 5663,4 & 379,9 & 2151,8 \\
\hline Madeira & 41059,9 & 417,1 & 17127,7 \\
\hline Raízes & 8621,3 & 400,7 & 3454,7 \\
\hline \multirow[t]{4}{*}{ Total } & & & 27676,3 \\
\hline & \multicolumn{2}{|c|}{ Povoamento com 6 anos de idade } & \\
\hline & \multirow[t]{2}{*}{ Biomassa $\left(\mathrm{kg} \mathrm{ha}^{-1}\right)$} & \multicolumn{2}{|c|}{ Carbono orgânico } \\
\hline & & $\mathrm{g} \mathrm{ha}^{-1}$ & $\mathrm{~kg} \mathrm{ha}^{-1}$ \\
\hline Folhas & 7579,0 & 451,9 & 3425,0 \\
\hline Galhos mortos & 3352,6 & 410,4 & 1375,8 \\
\hline Galhos vivos & 16227,9 & 387,7 & 6291,2 \\
\hline Casca & 7850,0 & 382,8 & 3005,4 \\
\hline Madeira & $90,976,3$ & 402,6 & 36631,5 \\
\hline Raízes & 12664,8 & 393,4 & 5019,9 \\
\hline Total & & & 55748,8 \\
\hline
\end{tabular}

A literatura mostra que o teor dos nutrientes nas folhas das árvores é influenciado por diversos fatores, como: condições de sítio, idade e posição das folhas na copa, época do ano. O elevado teor de carbono orgânico nas folhas de ambos os povoamentos de A. mearnsii, torna este componente da biomassa um grande reservatório, embora represente um pequeno percentual em relação a biomassa total das ár- vores. Nas folhas encontra-se a maioria das células vivas, que tendem acumular maiores quantidades de nutrientes em função dos processos transpiração e fotossíntese (KOZLOWSKI et al., 1991; KOZLOWSKI ; PALLARDY, 1996).

A produção de biomassa total é maior no povoamento com 6 anos de idade, isto é, o drobro em relação ao povoamento mais jovem, resultando em maior quantidade de carbono or- 
gânico retido por hectare $(50,4 \%$ a mais de carbono orgânico em relação ao povoamento com 4 anos de idade) (Tabela 4). Essa observação também foi verificada em Mimosa scrabella, na qual as árvores dos povoamentos mais velhos apresentaram maiores quantidades de biomassa de carbono, fato esse que está relacionado, conforme Rochadelli (2001), com o diâmetro e a presença de uma maior produção de biomassa de galhos.

A. mearnsii, com 6 anos de idade, fixou 53\% a mais de carbono orgânico na madeira em relação ao povoamento, com 4 anos de idade. Porém, conforme Ciesla apud Carpanezzi (2000), uma floresta de Acacia nilótica, com rotação de 10-15 anos, consegue reter na biomassa acima do solo $12-17 \mathrm{Mg}^{\mathrm{h}} \mathrm{H}^{-1}$ carbono orgânico.

O teor de carbono orgânico na madeira, para ambas as idades, foi semelhante em torno de 40\% (Tabela 4). Segundo Klock (1995), a composição química da madeira é considerada semelhante nas diversas espécies lenhosas. Aceita-se que as madeiras possuem, em geral, aproximadamente $50 \%$ de carbono.

A percentagem de biomassa carbono na biomassa, para ambos os povoamentos, foi praticamente igual, sendo $40,3 \%$ para o povoamento com 4 anos de idade e $40,5 \%$ para o povoamento com 6 anos de idade. Em estudo realizado por Rochadelli (2001), com Mimosa scabrella, a biomassa de carbono de cada árvore individual apresentou valores que variam entre 40 e $45 \%$, valores bem próximos daqueles indicados na literatura. O teores de carbono possuem, conforme o autor acima, uma tendência de acréscimos ao longo das árvores dominadas, vindo a diminuir novamente nas árvores dominantes.

\section{Conclusões}

Este estudo motra que:

O estoque de carbono total no solo, até 1,0 m de profundidade, foi similar nos povoamentos de A. mearnsii com 4 e 6 anos de idade.

A quantidade de carbono orgânico na serapilheira, bem como na biomassa total do povoamento de $A$. mearnsii, com 6 anos de idade, foi mais de $50 \%$ em relação ao povoamento com 4 anos de idade.
Povoamentos de A. mearnsii retêm grandes quantidades de carbono orgânico em sua biomassa, principalmente na madeira, comprovando a sua importância na capacidade de fixação do carbono.

\section{Referências}

BALBINOT, R.; SCHUMACHER, M. V.; HERNANDES, J. I. Carbono orgânico em uma floresta de Pinus taeda na Região de Cambará do Sul-RS. In: CONGRESSO E EXPOSIÇÃO INTERNACIONAL SOBRE FLORESTAS, 6. c2000. Porto Seguro. Resumos técnicos... Porto Seguro, BA. p. 56. 2000a.

CARPANEZZI, A. A. Benefícios indiretos da floresta. In: GALVÃO, A.P.M. (ed). Reflorestamento de propriedades rurais para fins produtivos e ambientais. Brasilília: Embrapa, 2000. 351p.

EMBRAPA. Centro Nacional de Pesquisa de Solos. Manual de métodos de análise de solo. Rio de Janeiro, 1997. 212p.

EMBRAPA. Centro Nacional de Pesquisa de Solos. Sistema brasileiro de classificação de solos. Brasília, 1999. 412p.

KLOCK, U. Química da madeira. FUPEF: Fundação de Pesquisas Florestais do Paraná, Curtiba., 1995. (Série Didática, n.4).

KOZLOWSKI, T. T.; KRAMER, P. J.; PALLARDY, S. G. The physiological ecology of woody plants. San Diego, USA: Academic, 1991. 657p.

KOZLOWSKI, T. T.; PALLARDY, S. G. Physiological of woody. 2. ed. San Diego, USA: Academic, 1996. 432p.

MOReno, J. A. Clima do Rio Grande do SuL. Porto Alegre: Secretaria da Agricultura, 1961. 41p.

O'CONNEL, A. M.; SANKARAN, K. V. Organic matter, accretion, de composition and mineralization. In: NAMBIAR, E. K. S.; BROWN, A. G. (eds) Management of soil nutrients and water in tropical plantations forests: ACIAR monograph, n.43, p.443-479, 571p. 1997.

PEREIRA, J. C.; CALDEIRA, M. V. W.; SCHUMACHER, M. V. et al. Estimativa do conteúdo de nutrientes em um povoamentos de Acacia mearnsii no Rio Grande do Sul - Brasil. Revista Árvore, Viçosa, 
v.24, n.2, p.193-199, 2000.

ROCHADELLI, R. A estrutura de fixação dos atómos de carbono em reflorestamento (Estudo de caso: Mimosa scrabella Bentham, bracatinga). 2001. 86f. Tese (Doutorado em Ciências Florestais) - Universidade Federal do Paraná, Curitiba.

SCHLESINGER, W. H. Carbon balance in terrestrial detritus. Annual Review of Ecology and Systematics, v.8, p.51-81, 1977.

SIMPSON, L. G.; BOTKIN, D. B. Vegetation, the global carbon cycle, and global measures. In: DUNNETTE, D.A.; O'BRIEN, R.J. (eds). The science of global change.

\section{Monography Series/American Chemical} Society, 1992. P.413-425.

TEDESCO, M. J.; GIANELLO, C.; BISSANI, C. A. et al. Análises de solo, plantas e outros materiais. Porto Alegre: Departamento de solos, UFRGS. 1995. 174p. (Boletim Técnico n. 5). 